\title{
WWOX, A NEW POTENTIAL TUMOR SUPPRESSOR GENE
}

\author{
Renata Hezova*, Jiri Ehrmann, Zdenek Kolar
}

\begin{abstract}
Department of Pathology \& Laboratory of Molecular Pathology, Faculty of Medicine and Dentistry, Palacky University, Hnevotinska 3, Olomouc 775 15, Czech Republic

e-mail:renatahezova@seznam.cz
\end{abstract}

Received: September 7, 2006; Accepted: February 1, 2007

Key words: WWOX/Tumor suppressor/Steroid dependent cancers/Nervous system

Background: WWOX (WW domain-containing oxidoreductase) gene, located on chromosome 16q 23.3-24.1 in the region recognized as the common fragile site FRA16D is considered to be a tumor suppressor gene involved in various cancers: breast, ovarian, prostate, esophageal, lung, pancreatic, gastric and hepatic. The aim of this study was to describe (i) putative protein interactions of WWOX (ii) the molecular mechanisms of tumor suppressor activity (iii) present an overview of WWOX in relation to nervous system and breast, prostate and ovarian cancers.

Methods and Results: WWOX expression is up-regulated in endocrine organs indicating its importance in these tissues. In many cancers WWOX expression is down-regulated and low WWOX expression is related to poor prognosis.

Conclusion: All the evidence suggest that WWOX can be considered as a new tumor suppressor gene and target for gene therapy due to the association of high WWOX expression with improved disease free survival.

\section{INTRODUCTION}

Chromosomal and genomic abnormalities affecting chromosome $16 \mathrm{q}$ have been frequently reported in cytogenetic and allelotypic studies of various epithelial tumors. Several authors have shown, that LOH of 16q, is often observed in breast carcinomas ${ }^{1-5}$. Other tumor types, such as prostate, ovarian, esophageal, lung, pancreatic, gastric and hepatic carcinomas, exhibit similar abnormalities of $16 \mathrm{q}^{6,7,8,14-19}$

Bednarek et al. ${ }^{8}$ cloned and described a gene mapping a genomic region of more than 1 million nucleotide bp located on chromosome 16q23.3-24.1, in a region recognized as the common fragile site FRA16D ${ }^{8}$. The gene was named WWOX (synonym name: fragile site FRA16D oxido-reductase, symbol: FOR). The WWOX gene is composed of nine exons and encodes a $46 \mathrm{kDa}$ protein that possesses two $\mathrm{NH}_{2}$-terminal WW domains and a short chain dehydrogenase domain (SDR) found in the SDR family of enzymes. WW domains are characterized by the presence of highly conserved proline and tryptophan residues $^{9-11}$ and occur in diverse types of proteins, such as Yes-associated protein, Nedd4, dystrophin, Rsp5, Pub1, FE65, Pin1, and FBPs. In common with the SH3 domain, the WW domain is characterized by its interaction with proline-containing ligands and mediating protein-protein interactions $^{12,13}$.

The SDR family of enzymes encompasses a wide spectrum of enzymes. One important group among SDR proteins is the family of hydroxysteroid dehydrogenases. These short-chain dehydrogenase/reductases are usually involved in the metabolism of steroid hormones such as androgens and estrogens. Further, WWOX expression is up-regulated in endocrine organs such as testis, ovary and breast, indicating the importance of WWOX in these tissues ${ }^{8}$.

A Drosophila model has been developed for the investigation of WWOX in vivo. The Drosophila DmWWOX orthologue shares $49 \%$ identity with human WWOX and has a conserved domain structure of tandem WW domains and oxidoreductase homology. DmWWOX null mutants are viable and fertile with no obvious phenotype. However, they show increased sensitivity to ionising radiation ${ }^{20}$. Protection from radiation can be restored by reintroduction of either Drosophila or human WWOX. The radiation sensitivity phenotype is similar to that previously observed for Drosophila mutants p53 (ref. ${ }^{21,22}$ ).

\section{PUTATIVE INTERACTIONS OF WWOX, MOLECULAR MECHANISMS OF TUMOR SUPPRESSOR ACTIVITY}

\subsection{WWOX-p73}

Aqeilan et al. ${ }^{38}$ showed that WWOX interacts via its first WW domain with the PPPY motif of p73. The tyrosine kinase, Src, phosporylates WWOX at tyrosine 33 in the first WW domain and enhances its binding to $\mathrm{p} 73$.

The p73 protein is a structural and functional homologue of the p53 tumor suppressor protein ${ }^{39,40}$. The $\mathrm{p} 73$ protein localizes in the nucleus, and not only recognises and binds to p53-responsive elements in the promoter regions of diverse p53-target genes such as $\mathrm{p} 21^{\text {Waf } 1 / \text { Cip } 1}$ and $\mathrm{Bax}^{41}$, but can also transactivate the transcription of these target genes. WWOX localizes to the cytoplasm, 
and consequently overexpression of WWOX causes the sequestration of $\mathrm{p} 73$ in cytoplasm and increases its proapoptotic activity ${ }^{38}$. It is likely that loss of WWOX in tumor cells may result in reduced p73 apoptotic activity in cytoplasm $^{38}$. Although mutations of $\mathrm{p} 73$ in cancer are not frequent ${ }^{41}$ and $\mathrm{p} 73$-defficient mice exhibit no predisposition to tumors ${ }^{43}$, several reports have established the role of p73 in apoptosis. Its modulation of p53 function may be important in malignity development ${ }^{42}$. Further studies will be necessary for evaluation of the biological consequences of this association in normal and cancer cells. Interestingly, human WWOX does not bind with $\mathrm{p} 53^{38}$ whereas the murine WWOX ortholog, Wox 1, interacts with p53 and Jnk1 under stress conditions ${ }^{45}$. Wox 1 also interacts synergistically with $\mathrm{p} 53$ during TNF-mediated apoptosis. This proapoptotic role of Wox 1 is negatively regulated by interaction with $\mathrm{JNK}^{44,45}$.

\subsection{WWOX-activating protein-2 $\lambda$ (AP-2 $\lambda$ )}

Aqeilan et al ${ }^{46}$ demonstrated a functional association between AP- $2 \lambda$ transcription factor and the WWOX protein. AP- $2 \lambda$ at $20 \mathrm{q} 13.2$ encodes a transcription factor and is frequently amplified in breast carcinoma. WWOX binds to the PPPY motif of AP- $2 \lambda$ via its first WW domain. Alterations of tyrosine 33 in the first WW domain of WWOX or the proline-rich motif in AP- $2 \lambda$ dramatically reduce this interaction. This shows that WWOX expression triggers redistribution of nuclear AP- $2 \lambda$ to the cytoplasm and hence suppresses its transactivating function. It is suggested that WWOX tumor suppressor protein inhibits AP- $2 \lambda$ oncogenic activity by its sequestering into the cytoplasm.

\subsection{WWOX-YES-associated protein (YAP)}

Yes-associated protein (YAP) also containing WW domains was shown to associate with p73 and enhance its transcriptional activity ${ }^{47}$. YAP competes with WWOX for interaction with ErbB-4 and modulates its transcriptional function. YAP interacts with ErbB-4 receptor tyrosine kinase and acts as a transcriptional coactivator of the $\mathrm{COOH}$-terminal fragment (CTF) of ErbB-4. Interaction of WWOX and ErbB-4 suppresses transcriptional coactivation of CTF by YAP in a dose-dependent manner. A mutant form of WWOX lacking interaction with ErbB-4 has no effect on ErbB-4 coactivation ErbB-4. Further, WWOX is able to inhibit coactivation of $\mathrm{p} 73$ by YAP. These data indicate that WWOX antagonizes the function of YAP by competing for its interaction with ErbB-4 and other targets and thus affects their transcriptional activity ${ }^{47}$.

\section{WWOX IN RELATION TO CANCER}

Low, undetectable expression or aberrant transcripts of WWOX have been reported in different types of cancer and several tumor cell lines of different origin ${ }^{23}$. The frequent deletion of WWOX in multiple tumors suggests that WWOX may act as a tumor suppressor gene ${ }^{46}$.

Bednarek et al. ${ }^{8}$ evaluated WWOX expression in normal human tissue by Northern blot analysis: high levels of WWOX RNA were detected in endocrine organs such as prostate, testis and ovary and weak levels were detected in small intestine, spleen and colon. Thymus, leukocytes and breast have to the contrary very low levels of WWOX RNA.

\subsection{WWOX and breast cancer}

In breast carcinoma, $\mathrm{LOH}$ on $16 \mathrm{q}$ was found in $50-$ $55 \%$ of early tumors ${ }^{19,22}$. Allelic imbalances of $16 \mathrm{q}$ have been reported in up to two-thirds of all sporadic breast carcinomas and have been detected even in the absence of other genetic alterations. Consequently, such losses have been considered very early events in breast carcinogen$\mathrm{esis}^{25}$. Some authors have described 16q loss as a discriminating factor among low-grade ductal invasive, tubular, and tubulolobular carcinomas ${ }^{26,27}$

Bednarek et al. ${ }^{28}$ showed that WWOX suppresses the tumorgenicity of breast cancer cells in vitro as well as in vivo. Stable expression of WWOX in MD-MB-435 and T47D breast carcinoma cells resulted in a dramatic reduction in colony number in soft agar. Increased WWOX expression after transfection of WWOX into MDA-MB435 breast cancer cells dramatically reduced tumorigenicity in nude mice. The breast cancer cell line expressing the highest levels of WWOX mRNA/protein are the ER+ (estrogen receptor positive) MCF7 cells. The ER- breast cancer lines MDA435 and MDA231 were found to have practically undetectable levels of WWOX protein. This suggests, there is a possible corelation between WWOX protein expression and ER status. Furthermore, other authors have also described a statistically significant correlation between WWOX expression and ER status in breast carcinomas $^{29,}{ }^{32}$. Nunez et.al. ${ }^{29}$ also studied PR (progesterone receptor) status, but due to the limited number of ER-PR+ breast carcinomas samples, they could not confirm that $\mathrm{PR}+$ status alone correlates with high WWOX expression.

Pluciennik et al. ${ }^{30}$ analyzed levels of WWOX expression in 132 cases of breast cancer. They showed that WWOX expression was higher in patients under 50, in ER and PR positive tumors and in tumors without lymph node metastasis. WWOX mRNA levels were also higher in tumors with a higher apoptotic index (Bcl2/Bax ratio).

Guler et al. ${ }^{32}$ evaluated WWOX expression immunohistochemically in 97 archived breast carcinoma specimens. Decreased WWOX expression was found in $63.2 \%$ of invasive tumors. Reduced WWOX staining was found more frequently in ER (-) or scanty positive tumors $(p=0.033)$ and WWOX expression in adjacent normal tissue was reduced in $32.9 \%$ of specimens, especially in patients with higher stage disease $(p=0.033)$. Highly reduced WWOX staining (> $10 \%$ ) in normal breast tissue was found in post-menopausal women or in breast cancer patients exposed to neoadjuvant chemotherapy, suggesting that WWOX expression may be associated with level of steroid hormone expression and can be affected by chemotherapy.

Guler et al. ${ }^{35}$ also compared expression of WWOX, ErbB2 and p53 in 44 pure ductal carcinoma in situ (DCIS) cases and 31 DCIS lesions adjacent to invasive 
tumors. Statistically significant loss of WWOX expression was found in $68,2 \%$ DCIS, followed $61,3 \%$ invasive tumors and 54,8 \% DCIS adjacent to invasive tumor.

Further studies will be necessary to prove whether loss of WWOX expression is an early causative event of breast cancer, for at least in a subset of tumors or, whether this event predominantly occurs during the progression of breast carcinomas.

\subsection{WWOX and prostate cancer}

Chang et al. ${ }^{34}$ postulated that progression from normal prostate to hyperplasia and non-invasive/invasive cancer stages positively correlate with up-regulation and activation of WWOX ${ }^{34}$.

Activated Cdc42-associated kinase (Ack1) primarily phosphorylates WWOX at tyrosine 287, resulting in WWOX being targeted for ubiquitination mediated degradation. Therefore WWOX protein can be activated or inactivated via ubiquitination, by tyrosine phosphorylation at either Tyr 33 or Tyr 287 respectively. Primary androgen-independent prostate tumors but not benign prostate showed increased tyrosine-phosporylated Ack1 and decreased WWOX. These results suggest that Ack1 stimulates prostate tumorigenesis at least in part by negative regulation of the tumor suppressor $\mathrm{WWOX}^{35}$.

\subsection{WWOX and ovarian carcinoma}

Nunez et al. ${ }^{36}$ analyzed the WWOX protein expression in normal ovaries and ovarian carcinomas $(n=444)$. Immunoblotting analysis of normal ovarian samples demonstrated consistently strong WWOX expression, whereas $37 \%$ of ovarian carcinomas showed reduced or undetectable WWOX protein expression In addition, immunohistochemistry of ovarian tissue showed either no or barely detectable levels of WWOX expression in $30 \%$ of tumors. The remaining ovarian carcinomas (70\%) were moderately to strongly positive for this protein. Significant loss of WWOX expression was detected in $70 \%$ of the mucinous and $42 \%$ of clear cell carcinomas. Reduced WWOX expression significantly correlated with clinical stage IV (FIGO - International Federation of Gynecology and Obstetrics $)(p=0.007)$, negative progesterone receptor $(P R)$ status $(p=0.008)$ and shorter overall survival $(p=0.03)$. Association with ER status was not significant.

\subsection{WWOX and other carcinomas}

Kuroki et al. ${ }^{37}$ showed similar tumor suppressor effect of WWOX in AsPc1 and Panc1 pancreatic carcinoma cells.

Allelic losses and homozygous deletions at the FRA16D common fragile site were also reported in gastric cancer ${ }^{50}$. Kuroki et al. ${ }^{51}$ examined 81 primary gastric adenocarcinomas immunohistochemically for WWOX protein expression and found a lack of WWOX protein expression in $65 \%$. They also found a significant correlation between WWOX expression and tumor histological grade, with higher-grade tumors being significantly more likely to be negative for Wwox protein expression.

Several reports have described the frequent allelic losses on chromosome 16q23-24 around the FRA16D locus in hepatocellular carcinoma ${ }^{16}$. Park et al. ${ }^{52}$ found that loss of the DNA copy-number confined to 16q23 was detected by comparative genomic hybridization in several hepatocellular carcinoma cell lines, and that WWOX protein expression was absent or diminished in $72 \%$ of cell lines.

The WWOX gene is also altered in lung cancer ${ }^{53}$. In nonsmall cell lung cancer, transcripts lacking WWOX exons were detected in $26 \%$ of tumors and in five of eight cell lines ${ }^{55}$. WWOX allele loss occurred in $37 \%$ of tumors, and the promoter was hypermethylated in $62.5 \%$ of squamous cell lung carcinomas ${ }^{17,54}$ Fabri et al. ${ }^{53}$ examined the tumor suppressor function of WWOX in preclinical lung cancer models. They performed restoration of WWOX levels with the help of adenovirus infection in endogenous WWOX protein-negative cell lines (A549, H460, and H1299). Restored expression of WWOX caused dramatic suppression of tumorigenicity of A549, H460, and H1299 cells in nude mice. A549 and H460 cells lines underwent apoptosis through activation of the intrinsic apoptotic caspase cascade after WWOX expression.

\section{WWOX AND THE NERVOUS SYSTEM}

\subsection{The role of Wox1 (murine homolog of human} WWOX) in the developing nervous system

Hyaluronidases involved in murine embryonic development such as PH-20, Hyal-1 and Hyal-2 induce expression of Wox1. Wox1 significantly down-regulates the apoptotic

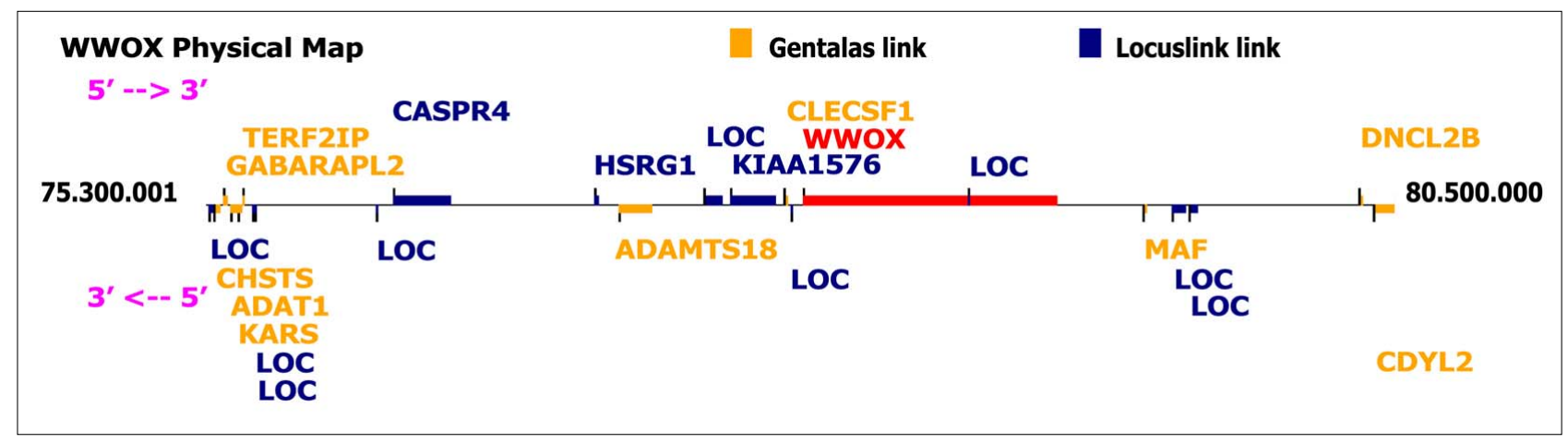

Fig. 1. Physical map of WWOX gene. 
inhibitors Bcl-2 and Bcl-xL, and up-regulates p53 expression and TNF cytotoxicity ${ }^{44}$. Chen et al. ${ }^{49}$ also reported, that WOX1 is expressed in the developing murine nervous system. WOX1 is differentially expressed during various stages of brain development. High levels of WOX1 protein have been observed in neural crest-derived structures such as cranial and spinal ganglia, pigmented skin cells, suggesting a potential role of WOX1 in promoting neuronal differentiation and maturation.

\subsection{WWOX and neurodegenerative diseases}

WWOX is likely involved in the pathogenesis of Alzheimer's disease (AD) and other neurodegenerative diseases involving neurofibrillary tangles. WWOX interacts with Tau proteins. Tau is a microtubule-associated protein that occurs mainly in neurons and is involved in neurite extension and maintenance. Although tau is a highly soluble protein and has a natively unfolded structure, it generates insoluble and hyperphosphorylated aggregates in Alzheimer's disease, which is characterized by degenerating neurons. WWOX binds Tau via its $\mathrm{COOH}$-terminal short-chain alcohol dehydrogenase/reductase domain and most likely prevents $17 \beta$ estradiol and enzyme- mediated Tau phosporylation in vivo. Down-regulation of WWOX in AD neurons of hippocampi induces Tau hyperphosporylation in vivo, leading to the formation of neurofibrillary tangles (NFTs) which characterize Alzheimer's disease. This suggests a protective role of WWOX in neurodegenerative disseases ${ }^{48}$.

\section{CONCLUSION}

Loss of WWOX expression is associated with a number of cancers including breast, ovary, testis, prostate, lung, pancreas, gastric and hepatocellular carcinomas. The role of WWOX has not yet been studied in brains tumors. In our earlier study we determined androgen receptor expression in brains tumor cell lines. It would be interesting to analyse, whether the WWOX plays a role in brain tumors.

The association of high WWOX expression to improved disease free survial, means that WWOX can be considered as a tumor suppresor gene and a new target for gene therapy ${ }^{30}$.

\section{ACKNOWLEDGEMENTS}

This work was partly supported by MSM 6198959216 and IGA MZ CR 1A/8244-3.

\section{REFERENCES}

1. Sato T, Tanigami A., Yamakawa K, Akiyama F, Kasumi F, Sakamoto G, Nakamura Y. (1990) Allelotype of breast cancer: cumulative allele losses promote tumor progression in primary breast cancer. Cancer Res 1990; 50:7184-7189.

2. Tsuda H, Callen D, Fukutomi T, Nakamura Y, Hirohashi S. Allele loss on chromosome 16q24.2-qter occurs frequently in breast can- cer irrespectively of differences in phenotype and extent of spread. Cancer Res 1994; 54:513-517.

3. Cleton-Jansen A., Moerland E., Kuipers-Dijkshoorn N, Callen D, Sutherland G, Hansen B, Devilee P, Cornelisse C. At least two different regions are involved in allelic imbalance on chromosome arm 16q in breast cancer. Genes Chromosomes Cancer 1994; 9:101-107.

4. Aldaz CM, Chen T, Sahin A, Cunningham J, Bondy M. Comparative allelotype of in situ and invasive human breast cancer: high frequency of microsatellite instability in lobular breast carcinomas. Cancer Res 1995; 55:3976-3981.

5. Chen T, Sahin A, Aldaz C. Deletion map of chromosome 16q in ductal carcinoma in situ of the breast: refining a putative tumor suppressor gene region. Cancer Res 1996; 56:5605-5609.

6. Carter BS, Ewing CM., Ward WS, Treiger BF, Aalders TW, Schalken JA., Epstein JI., Isaacs WB. Allelic loss of chromosomes 16q and 10q in human prostate cancer. Proc. Natl. Acad. Sci. 1990; 87:8751-8755.

7. Nishida N, Fukuda Y, Kokuryu H, Sadamoto T, Isowa G, Honda $\mathrm{K}$, Yamaoka Y, Ikenaga M, Imura H, Ishizaki K. Accumulation of allelic loss on arms of chromosomes 13q, 16q and 17p in the advanced stages of human hepatocellular carcinoma. Int. J. Cancer 1992; 51:862-868

8. Bednarek AK, Laflin KJ, Daniel RL, Liao Q, Hawkins KA, Aldaz CM. WWOX, a Novel WW Domain-containing Protein Mapping to Human Chromosome16q23.3-24.1, a Region Frequently Affected in Breast Cancer, Cancer Res 2000; 60:2140-2145.

9. Bork P, Sudol M. The WW domain: a signalling site in dystrophin. Trends Biochem. Sci. 1994; 19:531-533.

10. Andre B, Springael JY. WWP, a new amino acid motif present in single or multiple copies in various proteins including dystrophin and the SH3-binding Yesassociated protein YAP65. Biochem. Biophys. Res. Commun. 1994; 205:1201-1205.

11. Hofmann K, and Bucher P. The FHA domain: a putative nuclear signalling domain found in protein kinases and transcription factors. Trends Biochem. Sci. 1995; 20:347-349.

12. Ilsley JL, Sudol M, Winder SJ. The WW domain: linking cell signalling to the membrane cytoskeleton. Cell Signalling 2002; 14:183-189.

13. Macias MJ, Wiesner S, Sudol M. WW and SH3 domains, two different scaffolds to recognize proline-rich ligands. FEBS Lett 2002; 513:30-37.

14. Ried K, Finnis M, Hobson L, Mangelsdorf M, Dayan S, Nancarrow JK, Woollatt E, Kremmidiotis G, Gardner A, Venter D, Baker E, Richards RI. Common chromosomal fragile site FRA16D sequence: identification of the FOR gene spanning FRA16D and homozygous deletions and translocation breakpoints in cancer cells. Hum Mol Genet 2000; 9:1651- 63.

15. Paige AJ, Taylor KJ, Taylor C, Hillier SG, Farrington S, Scott D, Porteous DJ, Smyth JF, Gabra H, Watson JE. WWOX: a candidate tumor suppressor gene involved in multiple tumor types. Proc Natl Acad Sci 2001; 98:11417-22

16. Kuroki T, Trapasso F, Shiraishi T, Alder H, Mimori K, Mori M, Croce CM. Genetic alterations of the tumor suppressor gene WWOX in esophageal squamous cell carcinoma. Cancer Res 2002; 62:2258-60.

17. Yendamuri S, Kuroki T, Trapasso F, Henry AC, Dumon KR, Huebner K, Williams NN, Kaiser LR, Croce CM. WW domain containing oxidoreductase gene expression is altered in non-small cell lung cancer. Cancer Res 2003; 63:878-81.

18. Kuroki T, Yendamuri S, Trapasso F, Matsuyama A, Aqeilan RI, Alder H, Rattan S, Cesari R, Nolli ML, Williams NN, Mori M, Kanematsu T, Croce CM. The tumor suppressor gene WWOX at FRA16D is involved in pancreatic carcinogenesis. Clin Cancer Res 2004; 10:2459-65.

19. Aqeilan R, Kuroki T, Pekarsky Y, Albagha O, Trapasso F, Baffa R, Huebner K, Edmonds P, Croce CM. Loss of WWOX expression in gastric carcinoma. Clin Cancer Res 2004; 10:3053-8.

20. O'Keefe LV, Liu Y, Perkins A, Dayan S, Saint R, Richards RI. FRA16D common chromosomal fragile site oxido-reductase (FOR/WWOX) protects against the effects of ionizing radiation in Drosophila. Oncogene 2005; 24:6590-6596 
21. Sogame N, Kim M, Abrams JM. Drosophila p53 preserves genomic stability by regulating cell death. Proc Natl Acad Sci 2003; 100:4696-4701.

22. Lee JH, Lee E, Park J, Kim E, Kim J. Chung J. In vivo p53 function is indispensable for DNA damageinduced apoptotic signaling in Drosophila. FEBS Lett 2003; 550:5-10.

23. Paige AJ, Taylor KJ, Taylor C, Hillier SG, Farrington S, Scott D, Porteous DJ, Smyth JF, Gabra H, Watson JE. WWOX: a candidate tumor suppressor gene involved in multiple tumor types. Proc. Natl. Acad. Sci. 2001; 98:11417-11422.

24. Driouch K, Dorion-Bonnet F, Briffod M, Champeme MH, Longy M, Lidereau R Loss of heterozygosity on chromosome arm 16q in breast cancer metastases. Genes Chromosomes Cancer 1997; 19:185-191.

25. Buerger H, Otterbach F, Simon R. Different genetic pathways in the evolution of invasive breast cancer are associated with distinct morphological subtypes. J Pathol. 1999; 189:521-526.

26. Roylance R, Gorman P, Hanby A, Tomlinson I. Allelic imbalance analysis of chromosome $16 \mathrm{q}$ shows that Grade I and Grade III invasive ductal breast cancers follow different genetic pathways. J Pathol. 2002; 196:32-36.

27. Buerger H, Mommers EC, Littmann R, Simon R, Diallo R, Poremba C, Dockhorn-Dworniczak B, van Diest PJ, Boecker W. Ductal invasive G2 and G3 carcinomas of the breast are the end stages of at least two different lines of genetic evolution. J Pathol. 2001; 194:165-170.

28. Bednarek AK, Keck-Waggoner CL, Daniel RL, Laflin KJ, Bergsage PL, Kiguchi K, Brenner AJ, Aldaz CM. WWOX, the FRA16D gene, behaves as a suppressor of tumor growth. Cancer Res 2001; 61:8068 -73.

29. Nunez MI, Ludes-Meyers J, Abba MC, Kil H, Abbey NW, Page RE, Sahin A, Klein-Szanto AJ, Aldaz CM. Frequent loss of WWOX expression in breast cancer: correlation with estrogen receptor status, Cancer Research and Treatment 2005; 89:99-105. 30. Pluciennik E, Kusinska R, Potemski P, Kubiak R, Kordek R, Bednarek AK WWOX-the FRA16D cancer gene: Expression correlation with breast cancer progression and prognosis. Eur J Surg Oncol. 2006; 32(2), 153-7.

31. Wezel T, Lombaerts M, Roon EH, Philippo K, Baelde HJ, Szuha $\mathrm{K}$, Cornelisse CJ, Cleton-Jansen AM. Expression analysis of candidate breast tumour suppressor genes on chromosome 16q. Breast Cancer Research 2005; 7:R998-R1004.

32. Guler G, Uner A, Guler N, Han SY, Iliopoulos D, Hauck WW, McCue P, Huebner K. The Fragile Genes FHIT and WWOX Are Inactivated Coordinately in Invasive Breast Carcinoma. Cancer 2004; 100(8):1605-14.

33. Guler G, Uner A, Guler N, Han SY, Iliopoulos D, McCue P, Huebner K. Concordant loss of fragile gene expression early in breast cancer development. Pathology International 2005; 55: 471478.

34. Chang NS, Schultz L, Hsu LJ, Lewis J, Su M, Sze CI. 17beta-Estradiol upregulates and activates WOX1/WWOXv1 and WOX2/WWOXv2 in vitro: potential role in cancerous progression of breast and prostate to a premetastatic state in vivo. Oncogene 2005; 24(4), 714-23. 35. Mahajan NP, Whang YE, Mohler JL, Earp HS. Activated tyrosine kinase Ack1 promotes prostate tumorigenesis: role of Ack1 in polyubiquitination of tumor suppressor Wwox. Cancer Res. 2005; 65(22):10514-23

36. Nunez MI, Rosen DG, Ludes-Meyers JH., Abba MC, Kil H, Page R, Klein-Szanto AJ, Godwin AK, Liu J, Mills GB, Aldaz CM WWOX protein expression varies among ovarian carcinoma histotypes and correlates with less favorable outcome. BMC Cancer 2005; 5:64.

37. Kuroki T, Yendamuri S, Trapasso F, Matsuyama A, Aqeilan RI, Alder H, Rattan S, Cesari R, Nolli ML, Williams NN, Mori M,
Kanematsu T, Croce CM. The tumor suppressor gene WWOX at FRA16D is involved in pancreatic carcinogenesis. Clin Cancer Res 2004; 10:2459-65.

38. Aqeilan RI, Pekarsky Y, Herrero JJ, Palamarchuk A, Letofsky J, Druck T, Trapasso F, Han SY, Melino G, Huebner K, Croce CM. Functional association between Wwox tumor suppressor protein and p73, a p53 homolog. Proc Natl Acad Sci 2004; 101:4401-6.

39. Kaghad M, Bonnet H, Yang A, Creancier L, Biscan JC, Valent A, Minty A, Chalon P, Lelias JM, Dumont X, Ferrara P, McKeon F, Caput D. Monoallelically expressed gene related to p53 at 1p36, a region frequently deleted in neuroblastoma and other human cancers. Cell 1997; 90:809-819.

40. Jost CA, Marin MC, Kaelin WJ. P73 is a human p53-related protein that can induce apoptosis. Nature 1997; 389:191-194.

41. Melino G, De Laurenzi V, Vousden K H. p73: Friend or foe in tumorigenesis. Nat. Rev. Cancer 2002; 2:605-615.

42. Inoue T, Stuart J, Leno R, Maki CG. Nuclear import and export signals in control of the p53-related protein p73. J. Biol. Chem. 2002; 277:15053-15060.

43. Yang A, Walker N, Bronson R, Kaghad M, Oosterwegel M, Bonnin J, Vagner C, Bonnet H, Dikkes P, Sharpe A, McKeon F, Caput D. p73-deficient mice have neurological, pheromonal and inflammatory defects but lack spontaneous tumours. Nature 2000; 404:99_ 103.

44. Chang NS, Pratt N, Heath J, Schultz L, Sleve D, Carey GB, Zevotek N. Hyaluronidase induction of a WW domain containing oxidoreductase that enhances tumor necrosis factor cytotoxicity. J Biol Chem 2001; 276:3361-70.

45. Chang NS, Doherty J, Ensign A. JNK1 physically interacts with WW domaincontaining oxidoreductase (WOX1) and inhibits WOX1-mediated apoptosis. J Biol Chem 2003; 278:9195-202.

46. Aqeilan RI, Palamarchuk A, Weigel RJ, Herrero JJ, Pekarsky Y, Croce CM. Physical and Functional Interactions between the Wwox Tumor Suppressor Protein and the AP-2_ Transcription Factor. Cancer Res 2004; 64:8256-8261.

47. Aqeilan RI, Donati V, Palamarchuk A, Trapasso F, Kaou M, Pekarsky Y, Sudol M, Croce CM. WW domain-containing proteins, WWOX and YAP, compete for interaction with ErbB-4 and modulate its transcriptional function. Cancer Res. 2005; 65:6764-72.

48. Sze CI, Su M, Pugazhenthi S, Jambal P, Hsu LJ, Heath J, Schultz L, Chang NS. Down-regulation of WW Domain-containing Oxidoreductase Induces Tau Phosphorylation in Vitro. A potential role in Alzheimer's disease. J Biol Chem. 2004; 279(29):30498506.

49. Chen ST, Chuang JI, Wang JP, Tsai MS, Li H, Chang NS. Expression of WW domain-containing oxidoreductase WOX1 in the developing murine nervous system. Neuroscience 2004; 124:831-839.

50. Mangelsdorf M, Ried K, Woollatt E, Dayan S, Eyre H, Finnis M. Chromosomal fragile site FRA16D and DNA instability in cancer. Cancer Res 2000; 60:1683-9.

51. Kuroki T, Tajima Y, Furui J, Kanematsu T. Common Fragile Genes and Digestive Tract Cancers. Surg Today 2006; 36:1-5.

52. Park SW, Ludes-Meyers J, Zimonjic DB, Durkin ME, Popescu NC, Aldaz CM. Frequent downregulation and loss of WWOX gene expression in human hepatocellular carcinoma. Br J Cancer 2004; 91:753-9.

53. Fabbri M, Iliopoulos D, Trapasso F, Aqeilan R, Cimmino A, Zanesi N, Yendamuri S, Han S, Amadori D, Huebner K, Croce CM. WWOX gene restoration prevents lung cancer growth in vitro and in vivo. PNAS 2005; 25:15611-15616.

54. Iliopoulos D, Guler G, Han S Y, Johnston D, Druck T, McCorkell KA, Palazzo J, McCue PA, Baffa R, Huebner K. WWOX gene restoration prevents lung cancer growth in vitro and in vivo. Oncogene 2005; $24: 1625-1633$ 